\title{
Memoria larga en el número de horas de vuelo de aeronave de inteligencia militar de la Fuerza Aérea Colombiana
}

\section{Evidence of long memory in the number of flight hours for military intelligence aircraft in Colombian Air Force}

\author{
Diego Fernando Lemus Polanía ${ }^{a}$ \\ dflemusp@gmail.com \\ Maribel Tique \\ maribeltique@gmail.com
}

\author{
Harold Eraso ${ }^{\mathrm{b}}$ \\ hecjsem@gmail.com \\ Andrés Eduardo Peña ${ }^{\mathrm{d}}$ \\ sociologoandres@gmail.com
}

\begin{abstract}
Resumen
Actualmente, el Departamento de Planeación y Estadística de la Fuerza Aérea Colombiana (FAC) planifica el número mensual de horas de vuelo que tendrá cada una de sus aeronaves mediante el promedio de las horas que estuvieron estos equipos en el aire en el trimestre inmediatamente anterior. Debido a la inexactitud de los pronósticos actuales se presentan una serie de complicaciones a la hora de ejecutar el presupuesto requerido pues generalmente resulta insuficiente. En el presente trabajo se identifica un modelo $\operatorname{ARFIMA}(p, d, q)$ que permite pronosticar adecuadamente las horas de vuelo de la aeronave B-350 de la Fuerza Aérea Colombiana y que puede ser empleado por el alto mando militar para tomar decisiones administrativas acertadas en la planeación y uso mensual de esta aeronave.
\end{abstract}

Palabras clave: Series de tiempo de memoria larga, parámetro de diferenciación fraccional, modelo $\operatorname{ARFIMA}(p, d, q)$, pronóstico.

\footnotetext{
anvestigador USTAdistica

${ }^{\mathrm{b}}$ Especialista en estaística aplicada

${ }^{\mathrm{c}}$ Especialista en estaística aplicada

${ }^{\mathrm{d}}$ Especialista en estaística aplicada
}

Abstract

The Planning and Statistics Department determines for one period the number of hours of flight of every aircraft of the Colombian Air Force using a simple average of the observations in the previous period. Due to the inaccuracy of current forecasts, a great number complications in budget execution arises for being usually 
insufficient. In this paper an $A R F I M A(p, d, q)$ model was adjusted to predict adequately the number of flight hours for the FAC B-350 aircraft and can be used by the military high command to make appropriate planning and strategy decisions for that aircraft.

Keywords: Long memory time series, fractional differencing parameter, ARFI$M A(p, d, q)$ model, forecast.

\section{Introducción}

Desde la expedición de la ley arma de la aviación (31 de diciembre de 1919), la Fuerza Aérea Colombiana (FAC) ha trabajado para ejercer y mantener el dominio del espacio aéreo de nuestro país empleando una serie de equipos diseñados para el cumplimiento de diferentes tareas entre las que se resaltan: logística, inteligencia y combate. La FAC cuenta en la actualidad con más de 250 aeronaves que requieren de un presupuesto anual para su óptimo funcionamiento, el cual depende fundamentalmente del número de horas de vuelo pronosticadas para la siguiente vigencia (año fiscal), el número de pilotos disponibles y de los planes de mantenimiento por equipo.

Por tanto, al ser un factor crítico dentro del cumplimiento de su misión, la FAC proyecta anualmente el número de horas de vuelo que se necesitan para cada una de sus aeronaves en la siguiente vigencia fiscal. Actualmente se presentan problemas sistemáticos en la estimación de la cifra requerida por aeronave pues esta depende de factores exógenos a la planeación realizada por la institución como son las diferentes situaciones de orden público que se presentan en el país, situaciones económicas y políticas del estado.

El presente trabajo busca ilustrar el procedimiento de identificación y validación del modelo en series temporales que fue propuesto para capturar la dinámica del número de horas de vuelo en algunos equipos de inteligencia militar de la FAC, el cual permitirá al Departamento de Planeación y Estadística de esta institución estructurar de manera más precisa los recursos financieros que serán empleados en las operaciones militares aéreas que se requieran para cumplir su misión.

El documento tiene la siguiente estructura: la primera sección está compuesta por el marco referencial y la teoría estadística considerada para la identificación del modelo de pronóstico del número de horas de vuelo de una aeronave de inteligencia militar (común para un conjunto de este tipo de equipos). En la segunda parte se presentan los resultados del modelo ajustado para la aeronave B-350 como referente del conjunto de equipos que tienen la misma dinámica en la variable de interés. Finalizando el documento se presentán las conclusiones y recomendaciones del trabajo. 


\section{Antecedentes}

La inteligencia aérea es la capacidad integrada a recolectar, procesar, explotar y difundir información precisa y oportuna para planear y conducir operaciones militares exitosas. La búsqueda de la información, referente a la inteligencia militar, se realiza empleando principalmente aeronaves (Manual de operaciones Aéreas, 2005). Actualmente la FAC cuenta con una serie de equipos diseñados para el cumplimiento de esta tarea; aeronaves que requieren de insumos, mantenimiento preventivo y combustible para su normal funcionamiento.

En ese sentido la variable de interés para la FAC está compuesta por dos factores: mantenimiento y combustible. La etapa de mantenimiento consiste en garantizar la cantidad de repuestos necesarios para que un avión funcione correctamente, por tanto, los planes de mantenimiento preventivo están asociados directamente a la cantidad de horas que ha volado el equipo. Por el contrario, el combustible definido como la cantidad de libras o galones que gasta un equipo en una hora de vuelo es un factor decisivo en el éxito de las operaciones militares llevadas a cabo por la FAC; por lo general este dato es suministrado por la casa fabricante de los aviones. En este momento, la FAC mide el número de horas de vuelo de sus aeronaves empleando el software denominado "Sistema Integrado de Información Operacional de Comando y Control" (SIIOC2).

Hoy por hoy, el Departamento de Planeación y Estadística de la FAC es el encargado de pronosticar el número de horas de vuelo de cada aeronave de la institución, la metodología empleada para tal fin es el promedio aritmético de las horas de vuelo realizadas por cada una de estas durante el trimestre previo a la solicitud de recursos económicos al gobierno. Debido a la inexactitud de los pronósticos actuales se presentan una serie de complicaciones a la hora de ejecutar el presupuesto requerido pues generalmente resulta insuficiente. Por lo anterior, la jefatura militar debe solicitar constantemente adiciones presupuestales que desencadenan procesos administrativos dispendiosos (tramitología) y, que en casos extremos pueden interrumpir las operaciones militares que se están realizando; lo que genera una desventaja estratégica en la lucha contra el narcotráfico y los ejércitos disidentes que operan en el país.

\section{Procesos de memoria larga}

En Palma (2007) y Lemus \& Castaño (2013) se define la propiedad de memoria larga de una serie temporal a partir del comportamiento límite de su función de autocovarianza. Puntualmente, un proceso estacionario $\left\{Y_{t}: t \in \mathbb{Z}\right\}$ tiene memoria larga si sus autocovarianzas muestrales $\left(\gamma_{k}\right)$ no son absolutamente sumables, es decir, si

$$
\sum_{k=-\infty}^{\infty}|\gamma(k)|=\infty .
$$


En Granger \& Joyeux (1980), Beran (1993) y Castaño et al. (2008) se pueden encontrar definiciones alternativas de los procesos de memoria larga. Por ejemplo, se puede afirmar que una serie de tiempo estacionaria $\left\{Y_{t}: t \in \mathbb{Z}\right\}$ con densidad espectral $f(\lambda)$ en la frecuencia $\lambda$ tiene memoria larga si, para una constante $C_{f}>0$ $\mathrm{y}$ un real $0<\beta<1$, se puede garantizar que

$$
f(\lambda) \sim C_{f}|\lambda|^{-\beta} \quad \text { cuando } \quad \lambda \rightarrow 0^{+},
$$

Por lo tanto, los procesos con la propiedad de memoria larga no están acotados en el dominio de la frecuencia para una vecindad alrededor de $\lambda=0$.

\section{Modelos ARFIMA(p,d,q): Definición y propie- dades}

Según Beran et al. (2013) un modelo $\operatorname{ARFIMA}(p, d, q)$ puede ser ajustado a un proceso estocástico $\left\{Y_{t}: t \in \mathbb{Z}\right\}$ si, y solamente si, este puede ser representado por medio de la siguiente expresión:

$$
\phi(L)(1-L)^{d} Y_{t}=\mu_{0}+\theta(L) a_{t}, \quad t=1, \ldots, T
$$

donde $\mathrm{L}$ denota el operador de rezagos, $\phi(L)$ y $\theta(L)$ son el polinomio autorregresivo y el polinomio de media móvil, respectivamente. Las $q+p$ raíces de los polinomios no deben ser comunes y deben estar fuera de círculo unitario. $(1-B)^{d}=\sum_{k=0}^{\infty}\left(\begin{array}{l}d \\ k\end{array}\right)(-B)^{d}$ es el operador de rezago fraccional, $\mu_{0}$ es la parte determinística del proceso y $a_{t}$ es un proceso de ruido blanco con media cero y varianza finita $\sigma_{a}^{2}$. El parámetro de diferenciación fraccional $d$ determina el grado de memoria y de estacionariedad del proceso $Y_{t}$.

En ese sentido, si $d=0,\left\{Y_{t}\right\}$ en (3) corresponde a un proceso $A R M A(p, q)$ estacionario e invertible de memoria corta. Si $d \in \mathbb{Z}^{+},\left\{Y_{t}\right\}$ en (3) será un proceso no estacionario con $d$ raíces unitarias, es decir, un proceso $\operatorname{ARIMA}(p, d, q)$. Si $d \in \mathbb{R}-\mathbb{Z},\left\{Y_{t}\right\}$ en (3) será un proceso fraccionalmente integrado $\operatorname{ARFIMA}(p, d, q)$. Autores como Beran (1992), Baillie (1996) y Lee \& Schmidt (1996) presentan las propiedades más relevantes de proceso de ruido blanco fraccionalmente integrado -ARFIMA $(0$, d,0)-, las cuales se resumen a continuación:

- Si $0<d<1 / 2$, el proceso en cuestión es estacionario con memoria larga. Lo anterior implica que la densidad espectral se concentrará en las frecuencias bajas y no estará acotada en cero. Por el contrario, la función de autocorrelación será positiva y decrecerá de forma hiperbólica a una tasa aproximada $k^{2 d-1}$. 
- $\mathrm{Si}-1 / 2<d<0$, el proceso en cuestión es estacionario con memoria corta. En ese sentido, la densidad espectral estará dominada por las frecuencias altas y se anula en el origen. En este escenario, la función de autocorrelación será negativas y decrecerá de forma hiperbólica a la tasa enunciada previamente.

Hosking (1981) demostró que el valor absoluto de la función de autocorrelación del proceso ARFIMA(p,d,q) en el intervalo $-1 / 2<d<1 / 2$ tiene un comportamiento similar al del proceso $\operatorname{ARFIMA}(0, \mathrm{~d}, 0)$ y en ese sentido $\left\{Y_{t}\right\}$ en (3) corresponde a un proceso fraccionalmente integrado $\operatorname{ARFIMA}(\mathrm{p}, \mathrm{d}, \mathrm{q})$, estacionario e invertible -Baillie et al. (2002)-.

\section{Pruebas de memoria larga}

Una actividad rutinaria en el proceso de identificación del modelo más adecuado para estudiar la dinámica de una serie temporal consiste en identificar si sus autocorrelaciones muestrales presentan un grado extremo de persistencia o un patrón de decrecimiento exponencial mediante alguna de las pruebas de raíz unitaria - $I(0)$ vs $I(1)^{1}$ - tradicionales en la literatura. En Maddala \& Kim (1999) y Wei (2006) se presenta una descripción detallada de algunas de las pruebas raíz unitaria más tradicionales, a saber, la prueba ADF de Dickey \& Fuller (1979), la prueba de Phillips \& Perron (1988), la prueba KPSS de Kwiatkowski et al. (1992) y la prueba de Elliott et al. (1996).

Según Diebold \& Rudebush (1989) y Lee \& Schmidt (1996), la potencia de las pruebas de raíz unitaria es muy baja cuando, bajo la hipótesis alternativa se tiene un proceso fraccionalmente integrado -FI(d)-, situación que ha motivado el desarrollo de nuevos enfoques (nuevas pruebas) tanto en el dominio del tiempo como en el de las frecuencias para probar la existencia de memoria larga.

Palma (2007) manifiesta que el estadístico de rango reescalado de Hurst (1951), la prueba GPH de Geweke \& Porter-Hudak (1983), el estadístico de rango reescalado modificado de Lo (1991) y las pruebas de multiplicadores de lagrange (LM) de Robinson (1994) y Robinson (1995) son ampliamente utilizadas para probar la existencia de un raíz fraccional en procesos estacionarios e invertibles. Trabajos como los de Beran (1995), Baillie (1996) y Castaño et al. (2008) ofrecen una descripción detallada de las técnicas mencionadas anteriormente. Además, en Castaño et al. (2008) se propone una prueba para investigar la existencia de larga memoria basada en una aproximación autorregresiva finita del componente de corto plazo del proceso.

En el caso no estacionario, las propuestas de Lobato \& Robinson (1996), Tanaka (1999), Dolado et al. (2002), Mayoral (2007), Lobato \& Velasco (2007) y la prueba HML de Harris et al. (2008) son los métodos más relevante en la literatura relacionada. En Lemus \& Castaño (2013) se ofrece una descripción detallada

\footnotetext{
${ }^{1} \mathrm{I}(0)$ : Proceso estacionario de memoria corta vs $\mathrm{I}(1)$ :Proceso no estacionario de raíz unitaria
} 
de las técnicas mencionadas anteriormente y se propone una nueva prueba para investigar la existencia de raíces fraccionales en procesos no estacionarios.

\section{Análisis empírico del tiempo en operación diaria de una aeronave King Air B350}

Los datos utilizados en este estudio son observaciones diarias del número de horas de vuelo de una aeronave King Air B350 en operaciones de inteligencia militar estratégica $\left(Y_{t}\right)$ en el periodo comprendido entre el 01 de enero de 2011 hasta el 15 de agosto de 2014 para un total de 1323 observaciones. Los datos fueron obtenidos para el desarrollo del trabajo de grado de especialización propuesto por Tique et al. (2014) y se presentan en la Figura 1.

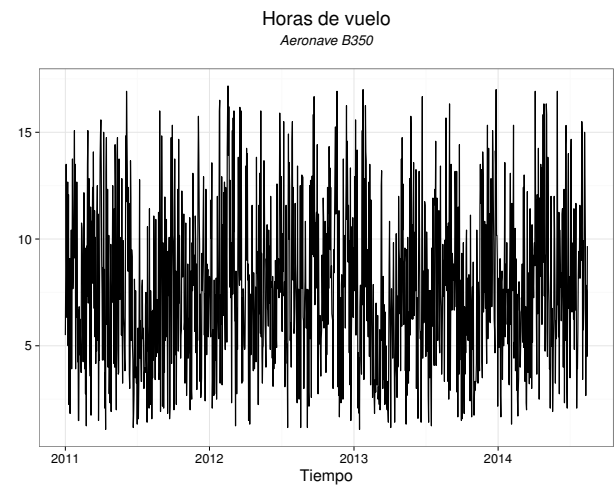

Figura 1: Serie - Aeronave B350

En este trabajo se determinó que la transformación Box-Cox más adecuada para estabilizar la varianza incondicional del proceso es la raíz cuadrada. A partir de este momento se trabajará sobre la serie transformada: $Z_{t}=\sqrt{Y_{t}}$, la cual se presenta en la Figura 2. 


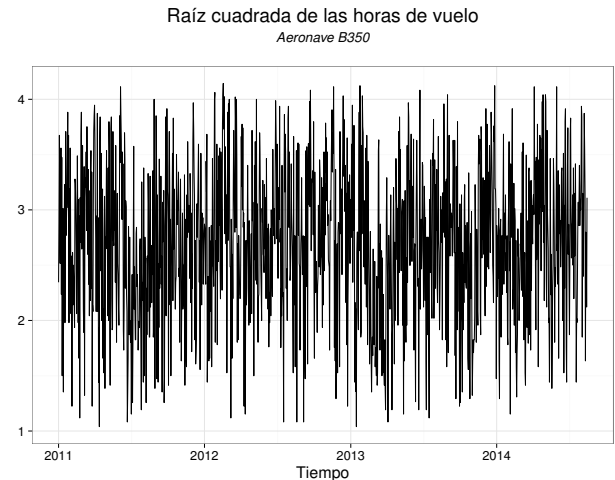

Figura 2: Serie transformada - Aeronave B350

En la Figura 3 se presenta el correlograma de la serie transformada.

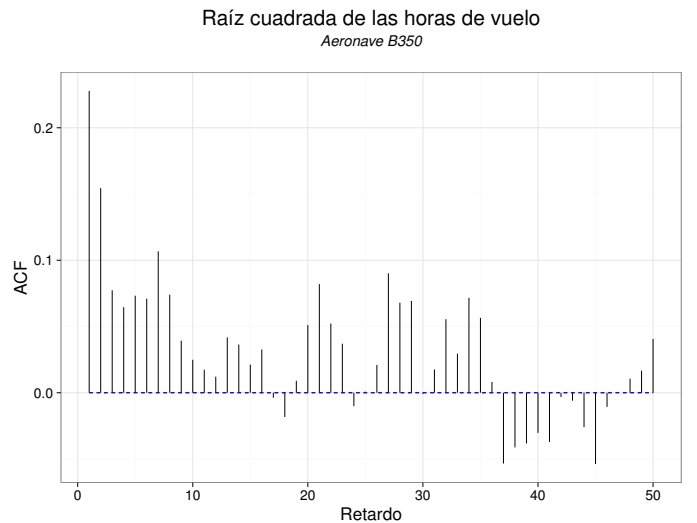

Figura 3: Función de autocorrelación

En la función de autocorrelación $(\mathrm{ACF})$ de la serie transformada se observa un decrecimiento que parece disminuir lentamente a cero pero sin presentar un grado extremo de persistencia, lo cual puede ser un indicio de que el proceso es posiblemente estacionario. En la Tabla 1 se presentan los resultados de las pruebas de Dickey-Fuller (ADF), de Phillips-Perron (PP), de Kwiatkowski et al. (1992) y la prueba ERS de Elliott et al. (1996) para determinar si hay evidencia o no de raíz unitaria en el proceso bajo estudio.

Comunicaciones en Estadística, diciembre 2018, Vol. 11, No. 2 
Tabla 1: Pruebas de raíz unitaria

\begin{tabular}{c|c|c|c|c}
\hline Pruebas de & Estadísticos & \multicolumn{3}{|c}{ Valores Críticos } \\
\cline { 3 - 5 } raíz unitaria & de prueba & $1 \%$ & $5 \%$ & $10 \%$ \\
\hline ADF & -20.15 & -3.43 & -2.86 & -2.57 \\
PP & -29.75 & -3.43 & -2.86 & -2.57 \\
KPSS & 0.12 & 0.74 & 0.46 & 0.35 \\
ERS & -6.97 & -2.57 & -1.94 & -1.62 \\
\hline
\end{tabular}

De los resultados presentados en la Tabla 1 se puede concluir que, para cualquier nivel de significancia nominal considerado $(90 \%, 95 \%$ y $99 \%$ de confianza) todas la pruebas de raíz unitaria rechazan su hipótesis nula ${ }^{2}$ al ser sus respectivos estadísticos de prueba menores que los valores críticos observados. Lo descrito anteriormente implica que no hay evidencia de raíz unitaria en la serie transformada $Z_{t}$. Resulta importante señalar que el modelo elegido para hacer las pruebas de raíz unitaria fue el siguiente

$$
Z_{t}=c+\phi Z_{t-1}+\epsilon_{t}
$$

donde $\epsilon_{t} \sim R B N\left(0, \sigma^{2}\right)$, el cual incluye una constante $c$ para capturar la media no nula bajo la hipótesis alternativa establecida en el siguiente contraste:

$$
H_{0}: \phi=1 \Rightarrow Z_{t} \sim I(1) \text { Sin deriva vs } H_{1}:|\phi|<1 \Rightarrow Z_{t} \sim I(0)
$$

\subsection{Pruebas de Raíz Fraccional Estacionaria}

En esta subsección se presentan los resultados de la pruebas empleadas en presente trabajo para probar la existencia de un raíz fraccional en procesos estacionarios e invertibles. Las pruebas consideradas fueron el rango reescalado de Hurst (1951) y la prueba del rango reescalado modificado de Lo (1991) en las cuales de define el siguiente contraste de hipótesis

$$
H_{0}: H=0.5 \text { vs } H_{a}: H \neq 0.5
$$

siendo $\mathrm{H}$ el coeficiente de Hurst. Bajo la hipótesis nula $(H=0.5)$ el proceso $Z_{t}$ corresponde a un proceso $A R M A(p, q)$ estacionario e invertible; bajo la alternativa $(0 \leq H \leq 1, H \neq 0.5)$ el proceso $Z_{t}$ corresponde a un proceso $\operatorname{ARFIMA}(p, d, q)$ estacionario e invertible. De forma similar a la prueba bilateral, se pueden realizar pruebas laterales para contrastar la hipótesis nula contra la alternativa donde el proceso $Z_{t}$ exhibe una fuerte reversión a la media y es antipersistente $\left(H_{a}\right.$ :

\footnotetext{
${ }^{2} H_{0}: Z_{t}$ es proceso no estacionario homogéneo con una raíz unitaria vs $H_{a}: Z_{t}$ es proceso estacionario sin raíz unitaria
}

Comunicaciones en Estadística, diciembre 2018, Vol. 11, No. 2 
$0 \leq H<0.5)$ o contra la alternativa de memoria larga sin reversión a la media $\left(H_{a}: 0.5<H \leq 1\right)$.

También se presentan los resultados de la prueba GPH de Geweke \& Porter-Hudak (1983), la prueba de Robinson (1995) y la prueba de Castaño et al. (2008) que consideran el siguiente contraste de hipótesis

$$
H_{0}: d=0 \text { vs } H_{a}: d \neq 0
$$

siendo d el parámetro de diferenciación fraccional. Bajo la hipótesis nula $(d=0)$ el proceso $Z_{t}$ corresponde a un proceso $\operatorname{ARMA}(p, q)$ estacionario e invertible; bajo la alternativa $(-0.5<d<0.5, d \neq 0)$ el proceso $Z_{t}$ corresponde a un proceso $\operatorname{ARFIMA}(p, d, q)$ estacionario e invertible. En las pruebas laterales se puede contrastar la hipótesis nula contra la alternativa donde el proceso $Z_{t}$ exhibe una fuerte reversión a la media y es antipersistente $\left(H_{a}:-0.5<d<0\right)$ o contra la alternativa de memoria larga $\left(H_{a}: 0<d<0.5\right)$. En la Tabla 2 se presenta el resumen de los resultados encontrados cuando se toma como hipótesis alternativa que la serie transformada del número de horas de vuelo de un aeronave King Air B350 presenta memoria larga.

Tabla 2: Pruebas de raíz fraccional

\begin{tabular}{c|c|c|c|c}
\hline Prueba & $\mathrm{H}$ & Error estándar & Estadístico de prueba & Valor P \\
\hline Hurst & 0.7042 & 0.0281 & 25.0802 & $2.6749 * 10^{-27}$ \\
Lo & 0.8808 & 0.0827 & 10.6450 & $2.3791 * 10^{-11}$ \\
\hline Prueba & $\mathrm{d}$ & Error estándar & Estadístico de prueba & Valor P \\
\hline GPH & 0.2615 & 0.0707 & 3.7005 & 0.0004 \\
Robinson & 0.3217 & 0.1065 & 3.0211 & 0.0021 \\
Castaño & 0.1873 & 0.0220 & 8.4966 & $<2.22 * 10^{-16}$ \\
\hline
\end{tabular}

Como los valores p de las pruebas de Hurst y Lo son menores al nivel de significancia nominal del $5 \%$ se rechaza hipótesis nula y se concluye que hay evidencia de memoria larga en la serie transformada del número de horas de vuelo de la aeronave B350. Las pruebas de Geweke \& Porter-Hudak (1983), la prueba de Robinson y la prueba de Castaño conducen al mismo resultado.

\subsection{Procedimiento de identificación y estimación}

En esta subsección se presenta el procedimiento propuesto en Castaño et al. (2008) para la identificación y estimación de un modelo adecuado para la serie transformada del número de horas de vuelo del equipo King Air B350 de la FAC. Siguiendo las especificaciones de los autores se aproxima el componente a corto plazo de la serie transfomada por medio de un proceso autorregresivo de orden $p^{*}=T^{1 / 4}=1407^{1 / 4} \simeq 6$ con el fin de realizar una estimación inicial 
del parámetro de diferenciación fraccional. Los resultados del modelo aproximado $\operatorname{ARFIMA}(6, d, 0)$ se presentan en la Tabla 3

Tabla 3: Modelo aproximado $\operatorname{ARFIMA}(6, d, 0)$

\begin{tabular}{c|c|c|c|c}
\hline Parámetro & Estimador & Error estándar & Estadístico de prueba & Valor P \\
\hline$\mu_{0}$ & 2.6981 & 0.0659 & 40.9269 & $<2 * 10^{-16}$ \\
$d$ & 0.1821 & 0.0077 & 23.735 & $<2 * 10^{-16}$ \\
$\phi_{1}$ & 0.0121 & 0.0285 & 0.425 & 0.671 \\
$\phi_{2}$ & 0.0244 & 0.0277 & 0.880 & 0.379 \\
$\phi_{3}$ & -0.0321 & 0.0276 & -1.163 & 0.245 \\
$\phi_{4}$ & -0.0221 & 0.0276 & -0.800 & 0.423 \\
$\phi_{5}$ & 0.0032 & 0.0275 & 0.115 & 0.908 \\
$\phi_{6}$ & 0.0013 & 0.0275 & 0.046 & 0.963 \\
\hline
\end{tabular}

En la Tabla 3 se observa que los coeficientes autoregresivos no son significativos (valores p mayores al nivel de significancia nominal del 5\%). Puesto que el modelo aproximado ajustado está sobreparametrizado se hace necesaria su reespecificación a un modelo aproximado que no incluya ningún coeficiente autoregresivo - $A R F I M A(0, d, 0)$ - obteniendo los resultados que se presentan en la Tabla 4:

Tabla 4: Modelo aproximado ARFIMA $(0, d, 0)$

\begin{tabular}{c|c|c|c|c}
\hline Parámetro & Estimador & Error estándar & Estadístico de prueba & Valor P \\
\hline$\mu_{0}$ & 2.6983 & 0.0692 & 39.0038 & $<2 * 10^{-16}$ \\
$d$ & 0.1873 & 0.0220 & 8.4966 & $<2 * 10^{-16}$ \\
\hline
\end{tabular}

De los resultados obtenidos se puede concluir que la estimación del parámetro de diferenciación fraccional d en el modelo aproximado resultó ser significativa para un nivel de significancia del $5 \%$ lo que parece indicar la existencia de memoria larga en la serie de tiempo bajo análisis. En la Tabla 5 se presentan los resultados de la prueba correlación serial de Ljung-Box para residuales modelo aproximado $\operatorname{ARFIMA}(0, d, 0)$ 
Tabla 5: Modelo aproximado ARFIMA(0,d,0)

\begin{tabular}{|c|c|c|}
\hline Estadístico de prueba & Grados de Libertad & Valor $\mathrm{p}$ \\
\hline 2.747 & 5 & 0.7389 \\
7.641 & 10 & 0.6639 \\
9.428 & 15 & 0.8541 \\
14.936 & 20 & 0.7801 \\
37.248 & 30 & 0.1703 \\
\hline
\end{tabular}

Los resultados de la prueba de Ljung-Box garantizan que el modelo $\operatorname{ARFIMA}(0, d, 0)$ es útil para capturar la correlación serial de corto plazo presente en la serie transformada y fraccionalmente diferenciada. Se ajustaron diferentes pruebas de normalidad sobre los residuos del modelo aproximado y se determinó que estos se ajustan a una distribución normal. Todas las pruebas diagnóstico se realizaron y se garantiza que el modelo aproximado es adecuado.

En el segundo paso del procedimiento se utiliza la estimación del parámetro de diferenciación fraccional del modelo aproximado para diferenciar la serie transformada del número de horas de vuelo del equipo King Air B350 de la FAC $W_{t}=(1-B)^{0.1873} Z_{t^{-}}$y posteriormente, identificar de los órdenes p y q de un modelo $A R M A(p, q)$ mediante la función de autocorrelación extendida (ESACF) y los criterios de selección de modelos AIC y BIC. Cabe resaltar que, para un proceso $\operatorname{ARMA}(\mathrm{p}, \mathrm{q})$, el vértice de un triángulo de ceros en la ESACF asintótica estará en la posición (p,q).

En la Tabla 6 se presenta la función de autocorrelación extendida obtenida sobre $W_{t}$; en esta se observa que el vértice de un triángulo de ceros se encuentra en la posición $(0,0)$, lo cual implica que el proceso $\operatorname{ARMA}(0,0)$ es consistente para modelar el comportamiento de corto plazo de la serie transformada fraccionalmente diferenciada.

Tabla 6: Resultado ESACF

\begin{tabular}{|c|ccccccccccc|}
\hline & \multicolumn{11}{|c|}{ MA } \\
\hline AR & 0 & 1 & 2 & 3 & 4 & 5 & 6 & 7 & 8 & 9 & 10 \\
0 & 0 & 0 & 0 & 0 & 0 & 0 & 0 & 0 & 0 & 0 & 0 \\
1 & $\mathrm{x}$ & 0 & 0 & 0 & 0 & 0 & 0 & 0 & 0 & 0 & 0 \\
2 & $\mathrm{x}$ & $\mathrm{x}$ & 0 & 0 & 0 & 0 & 0 & 0 & 0 & 0 & 0 \\
3 & $\mathrm{x}$ & $\mathrm{x}$ & $\mathrm{x}$ & 0 & 0 & 0 & 0 & 0 & 0 & 0 & 0 \\
4 & $\mathrm{x}$ & $\mathrm{x}$ & 0 & 0 & 0 & 0 & 0 & 0 & 0 & 0 & 0 \\
5 & $\mathrm{x}$ & $\mathrm{x}$ & 0 & $\mathrm{x}$ & $\mathrm{x}$ & 0 & 0 & 0 & 0 & 0 & 0 \\
\hline
\end{tabular}

Empleando los criterios de selección de modelos AIC y BIC se obtuvo el mismo modelo. Los resultados obtenidos indican que el proceso $A R F I M A(0, d, 0)$ es ade- 
cuado para modelar la serie transformada del número de horas de vuelo del equipo King Air B350. A continuación se presenta la verificación de que los supuestos básicos implícitos en el modelo se satisfacen. En la Figura 4 se presentan los residuales estandarizados del modelo ajustado

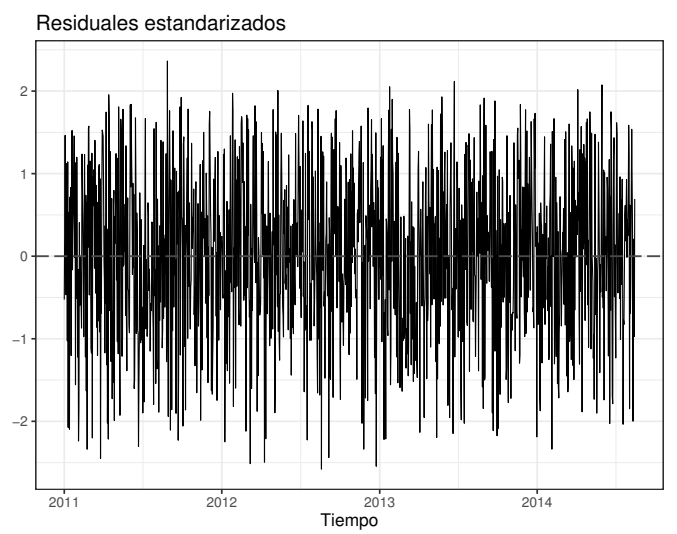

Figura 4: Residuales estandarizados - Modelo ARFIMA $(0, d, 0)$

en la Tabla 7 se presentan los resultados de algunas de las pruebas de normalidad sobre los residuales del modelo identificado.

Tabla 7: Pruebas de normalidad

\begin{tabular}{|c|c|c|c|}
\hline Prueba & & Estadístico de prueba & Valor $\mathrm{p}$ \\
\hline \multirow{2}{*}{ D'Agostino } & Omnibus & 0.696 & 0.7062 \\
& Sesgo & -0.821 & 0.4116 \\
& Curtosis & 0.147 & 0.8831 \\
\hline \multicolumn{2}{|c|}{ Shapiro-Wilk } & 0.989 & 0.3389 \\
Jarque-Bera & 0.867 & 0.5165 \\
\hline
\end{tabular}

Como el valor p de todas las pruebas consideradas es mayor al nivel de significancia considerado $(\alpha=0.05)$ no se rechaza la hipótesis nula, lo que permite afirmar que los residuales del modelo identificado se ajustan a una distribución normal. En la Figura 5 se presentan los resultados de la prueba de heterocedasticidad condicional de McLeod \& Li (1983) de la serie transformada. 


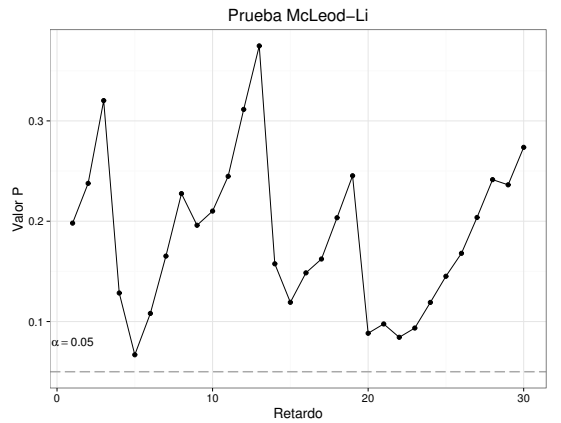

Figura 5: Prueba de McLeod - Li

En esta gráfica se observa que a un nivel de significancia aproximado de $\alpha=0.05$, se rechaza la hipótesis nula de no efecto ARCH. Los resultados obtenidos por las pruebas de correlación serial, de normalidad y de hetetocedasticidad condicional garantizan las validez del modelo $\operatorname{ARFIMA}(0, d, 0)$ ajustado $(1-B)^{0.1873} \sqrt{X_{t}}=a_{t}$ puede ser empleado para pronosticar valores futuros del número de horas de vuelo de la aeronave King Air B350.

\subsection{Pronósticos}

A la fecha, los métodos utilizados por la jefatura de operaciones aéreas para realizar los pronósticos de la cantidad de horas de vuelo necesarias por aeronave han sido prácticas basadas en la experiencia (Ad Hoc) o utilizando el promedio del último año; lo cual conlleva a pronósticos desfasados e inexactos que se reflejan en la ejecución de los planes operativos institucionales. Lo anterior, se ve reflejado en la escasez de material aeronáutico (repuestos) y en la insuficiente cantidad de combustible de aviación para cumplir con la misión asignada a la FAC por la constitución nacional.

En esta sección se presenta la comparación entre la precisión de los pronósticos del modelo ajustado con la precisión de los pronósticos del método empleado actualmente por la FAC mediante la metodología de validación cruzada. En el proceso de validación cruzada se utiliza el modelo $\operatorname{ARFIMA}(0, d, 0)$ ajustado en el período de entrenamiento comprendido entre el 01 de enero de 2011 hasta el 15 de junio de 2014 (1263 observaciones) para calcular los pronósticos del periodo comprendido entre el 16 de junio de 2014 hasta 16 de agosto de 2014; por otra parte, se empleó el promedio de horas de vuelo durante el último trimestre el 2014 como pronóstico actual de la FAC $\left(\bar{X}_{2014 t}=8.0328\right)$. Se emplearon el error cuadrático medio (MSE), la raíz cuadrada del error cuadrático medio (RMSE), el error medio absoluto (MAE), el error medio absoluto porcentual (MAPE)y el coeficiente de desigualdad de Theil como medidas de la precisión de los pronósticos obtenidos por los dos procedimientos. En la Tabla 8 se presentan los valores obtenidos para las medidas enunciadas en el párrafo anterior. 
252Diego Fernando Lemus Polanía, Harold Eraso, Maribel Tique \& Andrés Eduardo Peña

Tabla 8: Medidas de precisión de pronóstico

\begin{tabular}{c|c|c|c|c|c}
\hline Pronóstico & MSE & RMSE & MAE & MAPE(\%) & THEIL \\
\hline ARFIMA $(0, d, 0)$ & 14.07 & 3.75 & 3.03 & 16.67 & 0.06 \\
$\bar{x}_{2014}$ & 14.42 & 3.80 & 4.83 & 52.38 & 0.07 \\
\hline
\end{tabular}

Resulta importante resaltar que todas las medidas de precisión de pronóstico son menores para el modelo $\operatorname{ARFIMA}(0, d, 0)$ lo cual indica que los pronósticos realizados con este son más precisos que aquellos obtenidos con la metodología actual de la FAC. En la Figura 6 se presentan los pronósticos obtenidos por el modelo ajustado con tranformación directa y en la Figura 7 se presenta la restransformación propuesta por Guerrero \& Perera (2004) para eliminar el sesgo presente en esta tranformación.

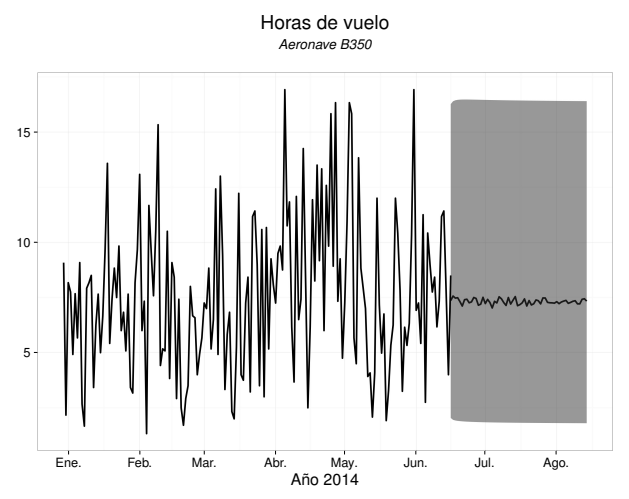

Figura 6: Pronósticos modelo $\operatorname{ARFIMA}(0, d, 0)$

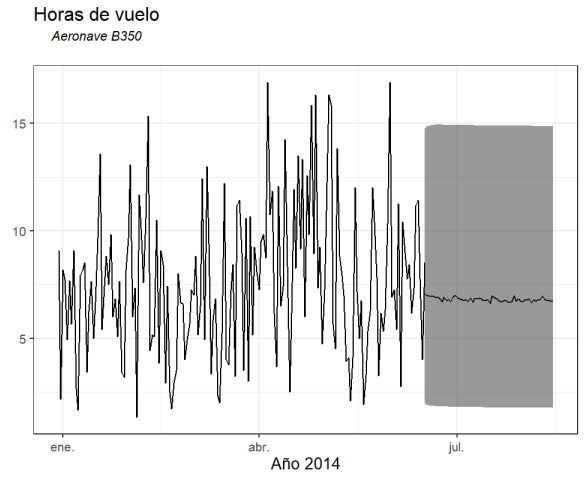

Figura 7: Pronósticos modelo $\operatorname{ARFIMA}(0, d, 0)$ con correción de transformación directa

Comunicaciones en Estadística, diciembre 2018, Vol. 11, No. 2 


\subsection{Conclusiones y recomendaciones}

Un buen modelo de pronóstico para el número de horas de vuelo de los equipos de la FAC puede ser empleado por el alto mando de la FAC como herramienta para tomar decisiones acertadas en la planeación y estrategia militar, sincronizar los procesos logísticos críticos y estandarizar el material requerido para el mantenimiento programado, es decir, podría impactar positivamente el análisis de todas las variables que hacen parte del planeamiento logístico que van desde la responsabilidad logística hasta el diseño de escenarios de horas a volar. Según los resultados obtenidos se pueden tener las siguientes conclusiones:

1. No hay evidencia de tendencia determinística ni de componente estacional para el número de horas de vuelo de la aeronave King Air B350 durante el periodo de tiempo considerado.

2. No hay evidencia de raíz unitaria en la serie, pero al emplear las pruebas de raíz fraccional consideradas se pudo establecer que la serie es estacionaria de memoria larga $(0<\hat{d}<0.5)$.

3. El modelo $A \operatorname{RFIMA}(0, d, 0)(1-B)^{0.1873} \sqrt{X_{t}}=a_{t}$ puede ser empleado para pronosticar valores futuros del número de horas de vuelo de la aeronave King Air B350 de la FAC.

4. Las medidas de precisión de pronóstico indican que las proyecciones del modelo $\operatorname{ARFIMA}(0, d, 0)$ son mucho más precisas que las predicciones actuales de la FAC.

como trabajo futuro, se considerará incluir la información sobre los periodos de mal tiempo climático en el país (por ejemplo, los periodos de invierno) y su posible efecto en el número de horas de vuelo de las aeronaves de la FAC.

Recibido: 2018-01-24

Aceptado: 2018-12-11

\section{Referencias}

Baillie, R. (1996), 'Long memory processes and fractional integration in econometrics', Journal of Econometrics 73(1), 5-59.

Baillie, R., Han, Y. \& Kwon, T. (2002), 'Further long memory properties of inflationary shocks', Southern Economic Journal 68(3), 496-510.

Beran, J. (1992), 'Statistical methods for data with long-range dependence', Statistical Science 7(4), 404-416.

Beran, J. (1993), 'Fitting long-memory models by generalized linear regression', Biometrika 80(4), 817-822.

Comunicaciones en Estadística, diciembre 2018, Vol. 11, No. 2 
Beran, J. (1995), 'Maximum likelihood estimation of the differencing parameter for invertible short and long memory autoregressive integrated moving average models', Journal of the Royal Statistical Society 57(4), 659-672.

Beran, J., Feng, Y., Ghosh, S. \& Kulik, R. (2013), Long-Memory Processes: Probabilistic Properties and Statistical Methods, 2th edn, Springer.

Castaño, E., Gómez, K. \& Gallón, S. (2008), 'Una nueva prueba para el parámetro de diferenciación fraccional', Revista Colombiana de Estadística 31, 67-84.

Dickey, D. \& Fuller, W. (1979), 'Distribution of the estimators for autoregressive time series with a unit root', Journal of the American Statistical Association 74(366), 427-431.

Diebold, F. \& Rudebush, G. (1989), 'Long memory and persistence in aggregate output', Journal of Monetary Economics 24, 189-209.

Dolado, J., Gonzalo, J. \& Mayoral, L. (2002), 'A fractional dickey-fuller test for unit roots', Econometrica 70(5), 1963-2006.

Elliott, G., Rothenberg, T. \& Stock, J. (1996), 'Efficient tests for an autoregressive unit root', Econometrica 64(4), 813-836.

Geweke, J. \& Porter-Hudak, S. (1983), 'The estimation and application of longmemory time series models', Journal of Time Series Analysis 4(4), 221-238.

Granger, C. \& Joyeux, K. (1980), 'An introduction to long-memory time series and fractional differencing', Journal of Time Series Analysis 1, 15-29.

Guerrero, V. M. \& Perera, R. (2004), 'Variance stabilizing power transformation for time series', Journal of Modern Applied Statistical Methods 3, 357-369.

Harris, D., McCabe, B. \& Leybourne, S. (2008), 'Testing for long memory', Econometric Theory 24(1), 143-175.

Hosking, J. R. M. (1981), 'Fractional differencing', Biometrika 68(1), 165-176.

Hurst, J. (1951), 'Long term storage capacity of reservoirs', Transactions of the American Society of Civil Engineers 116(1), 770-799.

Kwiatkowski, D., Phillips, P. C. B., Schmidt, P. \& Shin, Y. (1992), 'Testing the null hypothesis of stationarity against the alternative of a unit root: How sure are we that economic time series have a unit root?', Journal of Econometrics 54, 159-178.

Lee, D. \& Schmidt, P. (1996), 'On the power of the kpss test of stationary against fractionally-integrated alternatives', Journal of Econometrics 73(1), 285-302.

Lemus, D. \& Castaño, E. (2013), 'Prueba de hipótesis sobre la existencia de una raíz fraccional en una serie de tiempo no estacionaria', Lecturas de Economía 78(1), 151-184.

Comunicaciones en Estadística, diciembre 2018, Vol. 11, No. 2 
Lo, A. (1991), 'Long-term memory in stock market prices', Econometrica 59(5), 1279-1313.

Lobato, I. \& Robinson, P. M. (1996), 'Averaged periodogram estimation of long memory', Journal of Econometrics 73(1), 303-324.

Lobato, I. \& Velasco, C. (2007), 'Efficient wald tests for fractional unit roots', Econometrica 75(2), 575-589.

Maddala, G. \& Kim, I. (1999), Unit Roots, Cointegration, and Structural Change, 1th edn, Cambridge University Press.

Mayoral, L. (2007), 'Minimum distance estimation of stationary and nonstationary arfima processes', The Econometrics Journal 10(1), 124-148.

McLeod, A. I. \& Li, W. K. (1983), 'Diagnostic checking arma time series models using squared residual autocorrelations', Journal of Time Series Analysis 4, 269-273.

Palma, W. (2007), Long-Memory Time Series. Theory and methods, Jhon Wiley and Sons, Inc., Hoboken, New Jersey.

Phillips, P. \& Perron, P. (1988), 'Testing for a unit root in time series regression', Biometrika 75(2), 335-346.

Robinson, P. M. (1994), 'Efficient tests of nonstationary hypotheses', Journal of the American Statistical Association 89(428), 1420-1437.

Robinson, P. M. (1995), 'Log-periodogram regression of time series with long range dependence', The Annals of Statistics 23(3), 1048-1072.

Tanaka, K. (1999), 'The non-stationary fractional unit root', Econometric Theory 15(4), 549-582.

Tique, M., Peña, A. \& Castillo, H. (2014), 'Modelo de pronóstico de horas de vuelo para las aeronaves b-350 y sa2-37 de la fuerza aérea colombiana'.

Wei, W. (2006), Time Series Analysis : Univariate and Multivariate Methods, second edn, Pearson Education, Inc. 